\title{
Urinary tract infections in women: etiology and treatment options
}

\author{
This article was published in the following Dove Press journal: \\ International Journal of General Medicine \\ 19 April 201 I \\ Number of times this article has been viewed
}

\section{Daniele Minardi \\ Gianluca d'Anzeo \\ Daniele Cantoro \\ Alessandro Conti \\ Giovanni Muzzonigro}

Department of Clinical and Specialist Sciences, Urology, Polytechnic University of the Marche Medical School and United Hospitals, Ancona, Italy
Correspondence: Daniele Minardi Department of Clinical and Specialist Sciences, Urology, c/o Ospedali Riuniti, Via Conca 7I, Ancona 60126, Italy Tel +3907 I596 5667

Fax +3907 15963367

Email d.minardi@univpm.it
Abstract: Urinary tract infections (UTI) are common among the female population. It has been calculated that about one-third of adult women have experienced an episode of symptomatic cystitis at least once. It is also common for these episodes to recur. If predisposing factors are not identified and removed, UTI can lead to more serious consequences, in particular kidney damage and renal failure. The aim of this review was to analyze the factors more commonly correlated with UTI in women, and to see what possible solutions are currently used in general practice and specialized areas, as well as those still under investigation. A good understanding of the possible pathogenic factors contributing to the development of UTI and its recurrence will help the general practitioner to interview the patient, search for causes that would otherwise remain undiscovered, and to identify the correct therapeutic strategy.

Keywords: urinary tract infection, women, etiology, diagnosis, treatment

\section{Etiology}

Escherichia coli and Staphylococcus saprophyticus account for about $80 \%$ of community-acquired uncomplicated urinary infections (UTI), particularly in women under 50 years of age. ${ }^{1,2} \mathrm{E}$. coli is a Gram-negative commensal of the distal colon which also harbors other anaerobic bacteria, including Bacteroides and Bifidobacteria. ${ }^{3}$ Uropathogenic $E$. coli differs from intestinal pathogenic $E$. coli with regard to the presence of specific virulence factors. Among the various serotypes of E. coli, 01, 02, 04, 06, 07, 08, 016, 018, 025, and 075 are commonly recovered from patients with UTI. ${ }^{4}$ About $80 \%$ of uropathogenic E. coli express P fimbriae which anchor to the glycolipid of the outer membranes of urothelial cells localized in the kidney., $\mathrm{P}$ fimbriae are frequently associated with cases of acute pyelonephritis.

Another important virulence factor is Type 1 fimbriae. These are very important in the mechanism of bacterial adhesion to the uroepithelium. They are comprised of several subunits, the most important of which is an adhesion protein known as FimH which plays a principal role in the pathogenic mechanism of E. coli at the level of the urinary tract. It mediates both cellular invasion of E. coli and adhesion to mannose-containing glycoproteins. ${ }^{7,8}$ In vivo and in vitro studies have shown that the pathogenicity of $E$. coli is mainly related to mechanisms of colonization and invasion of the bladder epithelium and the ability to form intracellular bacterial communities. FimH enables uropathogenic $E$. coli to escape the innate immune response by internalization within urothelial cells, mediated by the activation of host signal transduction cascades via protein tyrosine kinases, phosphoinositide-3 kinase, and localized host actin cytoskeletal rearrangements. ${ }^{9}$ After invasion, E. coli is harbored within vesicles 
inside infected urothelial cells, ${ }^{10}$ from which it cannot be expelled. FimH also plays a role in the modulation of apoptosis in epithelial cells and in the mechanisms of cytoplasmic replication. Therefore, it is a very powerful factor in invasion and virulence. When FimH is not present, the uroepithelium is usually able to clear $E$. coli colonies via expulsion and mechanisms of the cellular immune response.

S. saprophyticus is a common UTI pathogen in younger women. It is reported to colonize the rectum and, to a lesser extent, the cervix and urethra in a small proportion of women. ${ }^{11}$ $E$. coli is the main organism responsible for nosocomial UTI, but other Gram-negative pathogens, including Pseudomonas spp, Enterobacter spp, Serratia spp, Citrobacter spp, and urease-producing Klebsiella spp, Proteus spp, Corynebacterium urealyticum, and Providencia spp are also involved in this type of infection. ${ }^{12}$ They are commonly involved in nosocomial UTI due to the inability of antibiotics to penetrate into the biofilm formed around and within the infectious stone. ${ }^{13}$ Also, Gram-positive bacteria, including Enterococcus spp and Staphylococcus spp, can cause nosocomial UTI due to selective pressure from the antimicrobial agents used in hospitalized patients. Anaerobic bacteria are also described in UTI, but their role is not well-defined. ${ }^{14}$

Also increasing are the numbers of fungal UTI, particularly those caused by Candida spp, and, to a lesser extent, by Aspergillus spp and Cryptococcus neoformans. In one study, positivity for Candida spp was found in 5\% of urine specimens from a general hospital and in 10\% from a tertiary-level care center. ${ }^{15}$ Most UTI caused by Candida spp are associated with the use of indwelling urinary devices, including Foley catheters, internal stents, and percutaneous nephrostomy drains. Diabetics are particularly prone to fungal UTI ${ }^{16}$ Normally this kind of infection is acquired by patients with severe neutropenia, drug abuse, recent surgery (chest, abdomen), and systemic infection.

\section{Risk factors}

\section{Immune response}

The principal mechanism of defense against infectious agents in the urinary tract lies in the innate immune response. Animal models have identified a subgroup of toll-like receptors as the primary effectors in the pathway of response to noxious agents in the urinary tract. ${ }^{17}$ The binding of antigenic determinants of pathogenic strains, as happens with $\mathrm{P}$ fimbriae and Type 1 fimbriae for uropathogenic E. coli, activates a signaling cascade, leading to the production of cytokines, including interleukin (Il)-6, IL-8, and tumor necrosis factor. ${ }^{18}$ The same mechanism is not activated against the determinants of nonpathogenic strains, raising the possibility that there are also control mechanisms activating a level of tolerance to symbionts. ${ }^{19}$ With regard to recurrence of UTI, a subgroup of toll-like receptors, ie, TLR-4, was investigated and shown to play an important role in the host response to uropathogens. Mice in experimental models and children not expressing TLR-4 have been shown to lack responsivity to uropathogenic $E$. coli strains, resulting in development of asymptomatic bacteriuria, while individuals with moderately reduced expression of TRL-4 tend to develop asymptomatic bacteriuria after a first symptomatic episode of UTI. ${ }^{20}$

After binding of uropathogens to the receptor, the main cytokine involved in the response is IL-8, which binds to the CXCR-1 receptor on the neutrophil plasma membrane. CXCR1 mediates the migration of uropathogens through the urothelial wall, leading to pyuria, ie, the macroscopic presence of pus in the urine. IL- 8 levels in the blood have been demonstrated to correlate positively with the number of neutrophils found in the urine during infection. ${ }^{21}$

CXCR1 has also been suggested to play a role in the susceptibility of individuals to recurrent UTI. In particular, the tendency of children with low levels of these receptors to develop recurrent pyelonephritis has been established. ${ }^{22,23}$ Polymorphisms of CXCR1 were recently demonstrated, and studies are underway to see whether these qualitative variants are related to susceptibility to recurrent UTIs, with the possibility of either genetically dominant or recessive transmission. ${ }^{24}$

\section{Anatomical structure}

Anatomical factors and alterations also play an important role in the pathogenesis of UTI in women. The shortness of the urethra, with its close relationship to the anus, makes it easy for bacteria to ascend in the urinary tract. In women, fecal-perineal-urethral contamination is the most probable explanation for infections caused by enteric bacteria, as demonstrated by experiments evaluating the genotype of E. coli strains causing UTI in women., ${ }^{4,25}$

In a case-control study, 100 women with recurrent UTI and 113 controls were investigated to determine whether there were differences in perineal anatomic measurements, postvoid residual urine volume, or in urine voiding characteristics. The distance from the urethra to the anus was significantly shorter in cases than in controls $(4.8 \mathrm{~cm}$ and $5.0 \mathrm{~cm}$, respectively, $P=0.03$ ). No other differences were identified between cases and controls. These data suggest that pelvic anatomical characteristics may play a role in predisposing young women to recurrent UTI, especially those who do not have exogenous risk factors for these infections. ${ }^{26}$ 


\section{Altered vaginal biota}

Lactobacilli are the dominant bacteria in the vaginal biota and possess antimicrobial properties that regulate other urogenital microbiota by maintaining an acidic $\mathrm{pH}$ in the vagina and producing hydrogen peroxide ${ }^{27}$ Incomplete cure and recurrence of genitourinary tract infection leads to a shift in the local flora from a predominance of lactobacilli to coliform uropathogens. In a study by Kirjavainen et al it was confirmed that, in bacterial vaginosis, the normal lactobacillus microbiota of the vagina shifts to a more mixed composition, which is also typical during disease-free periods in women prone to UTI. While the vaginal flora were not dominated by the most common uropathogens per se, they were likely to be defective in terms of resisting infection because of the lack of colonization resistance-enhancing properties conferred by lactobacillus accumulation. ${ }^{28}$ The use of lactobacillus-containing probiotics has been suggested for the treatment and prophylaxis of bacterial urogenital infection. However, the results of UTI prophylaxis studies using lactobacilli remain inconclusive. ${ }^{29}$ An alteration in vaginal flora is also observed during the postmenopausal period, because estrogens stimulate the proliferation of lactobacilli and reduce the local $\mathrm{pH}^{30}$

\section{Genetic factors}

Some studies suggest that a family history of UTI in a first-degree relative increases the risk of recurrent UTI and pyelonephritis in women. This supports the idea of a genetic influence on defense mechanisms in the urogenital tract. ${ }^{30}$

The development of a symptomatic UTI depends on the balance between pathogen virulence and the host response to the pathogen. Polymorphism of receptors involved in the inflammation process seems to be involved in the degree of susceptibility to developing symptomatic UTI. Some authors have studied genetic polymorphism in asymptomatic bacteriuria, a condition considered to be a risk factor for symptomatic urinary tract infections. ${ }^{31}$ As previously discussed, toll-like receptors are critical sensors of infection, and control many aspects of the host immune defense response. The toll-like receptor signaling pathway initiates a proinflammatory response. After binding to urothelial cell receptors and expression of virulence factors by uropathogens (eg, Type 1 and P fimbriae), toll-like receptors are activated. Subsequently, they activate the innate immune response, along with production of cytokines. ${ }^{18}$ Cytokines are essential to modulate the immune response. IL- 8 is involved in neutrophil recruitment and activation by binding to the CXCR1 receptor. ${ }^{32}$ In a cross-sectional analysis of asymptomatic bacteriuria in 1261 women aged 18-49 years, originally enrolled as participants in a population-based case-control study of recurrent UTI and pyelonephritis, Hawn et al found that TLR2 and CXCR1 polymorphisms were associated with asymptomatic bacteriuria, and a CXCR1 variant was associated with urinary CXCL-8 levels. These results suggest that genetic factors are associated with the early human bladder immune response prior to the development of symptomatic UTI. ${ }^{33}$ Other studies found an association between UTI in adults and TLR1, TLR4, and TLR5 polymorphism. ${ }^{34}$ These data support the idea of a genetic component for increased susceptibility to UTI in women.

\section{Blood groups}

Several lines of evidence have suggested the role of blood group antigens as susceptibility factors for UTIs. P fimbriae recognize Gal1-4Ga1 and Ga1Na-3Gal1-4Ga1 as receptors, which contain oligosaccharide sequences in the globo series of glycolipids. ${ }^{35}$ These are also human $\mathrm{P}$ and $\mathrm{ABO}$ blood group antigens. Individuals with the $\mathrm{P} 1$ antigen are more predisposed to invasion by $\mathrm{P}$ fimbriated microorganisms (mannose-resistant hemagglutination-positive strains). Patients with the negative P1 antigen express a smaller amount of receptors for P fimbriated E. coli, so that attachment during passage through the boundary layer seems to be more difficult. It could be concluded that the existence of the $\mathrm{P} 1$ antigen may be a gap in the protective mechanism and could indicate an increased susceptibility to UTI. ${ }^{36}$ Women with a history of recurrent UTI are three to four times more frequently found to be nonsecretor phenotypes of the $\mathrm{ABO}$ histo-blood group than women without such a history. ${ }^{37}$ Furthermore, uroepithelial cells from nonsecretor women show enhanced adherence of uropathogenic E. coli compared with secretor women. Recent data suggest that the biochemic explanation for increased adherence of $E$. coli to nonsecretor uroepithelial cells and their propensity to develop recurrent UTI may be the presence of a unique globo series of glycolipid receptors selectively expressed by nonsecretor epithelial cells that bind uropathogenic E. coli. ${ }^{38}$

\section{Pregnancy}

In a prospective study by Haylen et al 1140 women aged 18-98 years were examined for recurrent UTI in different physiologic and pathologic conditions. ${ }^{39}$ A correlation was observed between nulliparity and recurrent UTI, particularly in woman younger than 50 years. The authors suggested that stretch and relaxation of the birth canal caused by pregnancy and delivery may be beneficial in preventing recurrent UTI 
by reducing the frictional effect of intercourse. ${ }^{39} \mathrm{~A}$ reduced frequency of intercourse and number of sexual partners may also account for these findings. In this study, there was a positive relationship between recurrent UTI and a postvoid residual urine volume of $>30 \mathrm{~mL}$.

Pregnancy is associated with asymptomatic bacteriuria in $4 \%-7 \%$ of cases and pyelonephritis in $0.5 \%-2 \%{ }^{40}$ Many studies have reported that pyelonephritis is more common during the second half of pregnancy. ${ }^{41}$ This is thought to result from increasing mechanical compression by the enlarging uterus. A history of recurrent UTI, diabetes, and anatomical abnormalities of the urinary tract increase the risk of developing a UTI during pregnancy. ${ }^{42}$ One of the most important risk factors for symptomatic infection is asymptomatic bacteriuria. Asymptomatic bacteriuria is the main risk factor for development of acute pyelonephritis. ${ }^{43}$ During pregnancy, development of UTI is correlated with stasis of urine in the ureters, which impairs emptying of the bladder, with an increased postvoid residual urine volume, vesicoureteral reflux, and increased urinary $\mathrm{pH} .{ }^{44}$

\section{Menopause}

The incidence of UTI in women increases with advancing age. Bacteriuria occurs in about $10 \%-15 \%$ of women aged $65-70$ years and in $20 \%-50 \%$ of women aged over 80 years. ${ }^{45}$ These values are higher than the $5 \%$ rate of bacteriuria reported in premenopausal women. In a study conducted by Raz et $\mathrm{al}^{46}$ in a group of 149 postmenopausal women, a strong association was noticed between anatomical and functional alterations of bladder emptying and recurrent UTI. Inherited factors and a history of premenopausal UTI have also been suggested as predisposing factors for the development of recurrent UTI. After menopause, there is a significant reduction in estrogen secretion by the ovary, which is often associated with vaginal atrophy. Estrogens stimulate proliferation of Lactobacillus in the vaginal epithelium, causing reduction of vaginal $\mathrm{pH}$, thereby preventing vaginal colonization by Enterobacteriaceae. In addition, the absence of estrogens decreases the volume of the vaginal muscles, resulting in slackness of the ligaments holding the uterus, pelvic floor, and bladder, resulting in prolapse of the internal genitalia. A placebo-controlled, double-blind study ${ }^{47}$ demonstrated a correlation between reduced estrogenic hormone levels after menopause and the development of recurrent UTI. Application of topical vaginal estrogens markedly reduced the incidence of recurrent UTI in this study. In older institutionalized women, the main factors for development of recurrent UTI are deterioration in functional status and urethral catheterization. ${ }^{48}$

\section{Diabetes}

Diabetes is another condition correlated with the development of asymptomatic bacteriuria and recurrent UTI. ${ }^{49}$ In a multicenter study of 589 women aged 18-75 years and affected by either Type 1 or 2 diabetes, the risk of developing a symptomatic UTI was increased by the same risk factors as those in younger women (sexual intercourse and oral contraceptive use) for Type 1 diabetes and by the presence of asymptomatic bacteriuria for Type 2 diabetes. ${ }^{50}$ For Type 1 diabetes mellitus, risk factors for asymptomatic bacteriuria included a longer duration of diabetes, peripheral neuropathy, and microalbuminuria. For Type 2 diabetes, the risk factors were higher age, microalbuminuria, and a recent symptomatic UTI. ${ }^{40}$ The same study showed a weak correlation between increased risk of UTI and poor regulation of diabetes, presence of diabetic cystopathy, neuropathy, microalbuminuria, or macrovascular complications. Glycosuria does not seem to be a risk factor for the development of asymptomatic bacteriuria or symptomatic UTI in vivo. Neuropathy involving the urinary tract may be a potential mechanism that could increase the risk of UTI in diabetics, because it may result in dysfunctional voiding and urinary retention. However, efforts to determine a relationship between high postvoid residual urine and increased risk of UTI have been inconclusive. No relationship between diabetes and uropathogens has been observed. However, first episodes of asymptomatic bacteriuria seem to be less often caused by E. coli, and more often caused by Klebsiella and Enterococcus faecalis. This suggests that asymptomatic bacteriuria and symptomatic UTI may be different disease processes, and that bacterial colonization in the absence of symptoms differs in patients with diabetes. ${ }^{1}$

\section{Dysfunctional voiding}

A correlation has also been observed between recurrent UTI and dysfunctional voiding. Dysfunctional voiding is defined as abnormal bladder emptying in neurologically normal individuals, especially young women in whom there is increased external sphincter activity during voluntary voiding. The etiology is unknown. It is thought to be a learned behavior due to adverse pelvic conditions, eg, inflammation or trauma. ${ }^{51}$ It could also be the result of voluntary withholding of urine, or it could be a primary abnormality with detrusor instability and a dyssynergic sphincter. Association between dysfunctional voiding and recurrent UTI has been documented. ${ }^{52}$ Dysfunctional voiding may disrupt laminar urine flow through the urethra, causing a UTI as bacteria are transferred back from the meatus to the bladder as a result 
of the "milk-back" phenomenon. ${ }^{53}$ Dysfunctional voiding is investigated classically by the use of videourodynamic studies, ${ }^{54}$ but perineal ultrasound has recently been proposed as a more helpful investigation. ${ }^{55}$

\section{Intercourse-related issues}

Several behaviors seem to be correlated with the development of recurrent UTI in healthy premenopausal women. In a casecontrol multivariate analysis by Scholes et al independent risk factors for recurrent UTI included frequency of intercourse in the previous month, 12-month spermicide use, and a new sex partner in the previous year. Second in magnitude only to frequency of intercourse were two nonbehavioral risk factors that remained in the model, ie, age at first UTI being younger than 15 years and a history of UTI in the mother. ${ }^{56}$ No associations were found between history of recurrent UTIs and pre- and postcoital voiding patterns, frequency of urination, delayed voiding habits, wiping patterns, douching, use of hot tubs, frequent use of pantyhose or tights, or body mass index. In a review done in 2007, Farage et al reviewed the relevant research. The studies failed to find significant associations between the use of panty liners and recurrence of vulvovaginal infections, either bacterial or Candida-related. Furthermore, the studies analyzed tended to reject the theory that use of these devices is theoretically capable of influencing genital health, $\mathrm{pH}$, or wetness, making the individuals more subject to recurrences of infection. ${ }^{57}$ Also, the use of other tight underwear or clothes has not been demonstrated to correlate with higher rates of UTI.

\section{Management Antibiotic therapy}

Management of UTI in women depends on a number of factors. First, a distinction must be made between asymptomatic and symptomatic infection. Bacteriuria is common in women, and its prevalence increases with advancing age. Treatment for this condition is not always considered necessary but, in women, the possibility of pregnancy makes evaluation worthwhile, because asymptomatic bacteriuria is associated with an increased risk of pyelonephritis and adverse outcomes of pregnancy. ${ }^{58}$ However, a symptomatic urinary infection should always be treated because it is unlikely to resolve spontaneously.

Antibiotic therapy is an important part of the therapeutic strategy for UTI, although control of predisposing factors as far as possible and prophylaxis are also necessary, in order to achieve complete resolution of infection. A therapeutic algorithm for the treatment of symptomatic
UTI has been developed from evidence-based consensus by the International Consultation on Urologic Diseases, a summary of which is available in the European Association of Urology guidelines. ${ }^{59}$

The increased antibiotic resistance seen in recent years suggests that the choice of antibiotic should be guided by culture and sensitivity assays. However, an empirical approach is still considered valid for community-acquired UTI in the absence of complicating factors, eg, pyelonephritis, chronic infection, and atypical symptoms.

To date, given that E. coli is the most common uropathogen ${ }^{60}$ a first approach with single-dose fosfomycin trometamol $3 \mathrm{~g}$, pivmecillinam $400 \mathrm{mg}$ for three days, or nitrofurantoin macrocrystals $100 \mathrm{mg}$ twice daily for five days, is considered to be the first-line choices for empirical treatment of acute symptomatic uncomplicated UTI (both of the lower and upper urinary tract) in all European countries. ${ }^{61,62}$

Use of trimethoprim-sulfamethoxazole is nowadays limited because of widespread microbial resistance and should only be used in regions with a known low resistance rate $(<20 \%)$ and after culture and sensitivity assay ${ }^{63}$ However, in countries where resistance is low, trimethoprim-sulfamethoxazole can still be a valid first-line antibiotic, and in many countries where its use has been limited for years, in consideration of the evidence-based international guidelines, the resistance rate has receded and made possible the reintroduction of this combination as a therapeutic strategy.

Although quinolones have high efficacy against the main genitourinary microorganisms, particularly in vitro, clinical trials have failed to demonstrate their superiority in eradicating infections in comparison with other drugs. Moreover, higher rates of side effects in comparison with the aforementioned drugs and their lower tolerability limit the use of quinolones as second-line therapy. Furthermore, surveys conducted in many European countries have already shown high resistance rates in E. coli strains for nalidixic acid and its derivatives (over $10 \%$ and up to $32.6 \%$ in Hungary). ${ }^{64}$ The quinolones are primarily indicated at higher doses for treatment of pyelonephritis and more complicated infections when susceptibility testing is available. In case of known resistance to quinolones, aminoglycosides and carbapenem are the drugs of choice. In all cases of persistence of symptoms or early recurrence (within 2 weeks of the end of therapy), it is essential to choose another antibiotic based on culture and sensitivity assay.

There is particular concern about treatment of asymptomatic and symptomatic bacteriuria during pregnancy. UTI in pregnancy exposes both the mother and fetus to a higher 
risk of complications, and the choice of therapy is obviously more limited. Aminopenicillins are commonly used in pregnancy because they are not considered to be teratogenic. ${ }^{65}$ Amoxicillin is well-absorbed orally and is to date a mainstay of treatment. Cephalosporins have also generally been considered to be safe options. However, some concern has been raised about the use of single agents because of case reports of ceftriaxone use during pregnancy and the possible development of kernicterus. Similarly, teratogenicity with cefaclor, cefalexin, and cefradine was noted in a surveillance study of over 4000 Michigan Medicaid recipients exposed to these agents. ${ }^{66}$ Cotrimoxazole is to be avoided because of its teratogenic effects resulting from interference with the metabolism of folic acid.

\section{Antibiotic prophylaxis}

After a single episode of UTI has resolved, the next step is to consider factors that could trigger a recurrence. This is particularly important in women, in whom urinary retention is very common. According to the fecal-perineal-urethral hypothesis, uropathogenic organisms resident in the rectal flora represent the reservoir for ascending UTI in women. Several factors have been claimed to be responsible for this, both anatomical and psychosocial, but all possible factors should be taken into account.

In a study of 86 women with dysfunctional voiding and recurrent UTI, Minardi et al reported a reduction in the recurrence rate after pelvic floor re-education with biofeedback, supporting the hypothesis that emptying disorders play an important role in the recurrence of UTI in women. ${ }^{67}$ Costantini et al reported similar results using alpha-blockers in women with voiding problems. Thus, functional problems contributing to recurrence of urinary infections were also significantly reduced. ${ }^{68}$

When all attempts at modification of patient behavior and lifestyle have failed to resolve the problem of recurrence, it may be necessary to start antimicrobial prophylaxis. There are currently no guidelines by which to identify nonpregnant women who should receive antibiotic prophylaxis for UTI. Some authors suggest that two recurrences within 6 months after therapy or three episodes per year could be considered an indication to establish prophylaxis after treatment. However, the patient's discomfort about the prospect of recurrence of symptoms should be taken into account when choosing whether or not to start prophylaxis. Several kinds of prophylactic regimens, including continuous, intermittent, and postcoital administration, have proved to be useful in cases of recurrent uncomplicated cystitis. Reports from studies conducted with different regimens show that up to $95 \%$ of recurrences resolve after 6 months of therapy. ${ }^{69}$ The antibiotics of choice for this purpose are nitrofurantoin, fosfomycin trometamol, cotrimoxazole, cephalosporins, and quinolones, all at lower than therapeutic dosages. In all cases, a prophylactic regimen should be initiated only after complete eradication of the original infection is confirmed by a negative urine culture, performed 1-2 weeks after the conclusion of primary therapy.

\section{Nonantibiotic prophylaxis}

Because antibiotic resistance is steadily increasing, alternative or additional prophylactic strategies have been investigated. In particular, research on potential dietary supplementation has focused on cranberry (Vaccinium macrocarpon) juice and its extracts, the benefits of which for reducing UTI recurrences have been known for a long time. Many trials have demonstrated its beneficial effects in women suffering from recurrent UTI, and reduction of UTI recurrence during pregnancy has also been observed. The mechanism of action is related to the active metabolite of cranberry, ie, proanthocyanidin A, which has been shown to be able to inhibit Type P fimbria-mediated E. coli adhesion to urothelial cells and induce deformation in both antibioticsusceptible and antibiotic-resistant bacterial strains, thus preventing bacterial adhesion to the urothelial layer. ${ }^{70,71}$

In a randomized clinical trial, a dose-dependent reduction of bacterial adhesion in response to administration of cranberry juice identified the minimum daily dose of proanthocyanidin A able to reduce urinary E. coli concentrations significantly to be $36 \mathrm{mg}$. Further studies have been conducted in women, and most of them have showed a significant decrease in reinfection rates, with some suggesting superiority in comparison with antibiotic prophylaxis, although the level of evidence is still quite low. ${ }^{72}$

Studies aimed at identifying well-tolerated cranberry doses in different formulations are underway, and are demonstrating a significant improvement in overall quality of life for patients treated with prophylactic doses of cranberry. ${ }^{73}$ It should be borne in mind that not all studies have adequately demonstrated the prophylactic activity of cranberry against recurrence of UTI. A randomized trial conducted by Barbosa et al in a large number of female adolescents treated after a first episode of acute UTI failed to identify a beneficial effect of cranberry. These investigators suggested, as a possible explanation, that the principal active ingredient may lie in other components used as additives in cranberry juice. ${ }^{74}$ 
An important physiologically protective mechanism against UTI lies in the preservation of the equilibrium of the normal vaginal microbial population due to the presence of different Lactobacillus species. It has been observed that healthy proliferation of these microorganisms is able to prevent colonization by pathogens. In particular, in cases where physiological changes in mucosal trophism (reduction of vaginal glycogen and increase in the $\mathrm{pH}$ ) occur, recurrent UTI is likely. Based on this consideration, and on the fact that dysmicrobisms in the female genitalia also predispose to urinary infections, several studies have investigated if intravaginal Lactobacillus administration could prevent UTI.

Other studies in postmenopausal women have shown that intravaginal administration of estriol helps to re-establish the normal flora, thus playing a protective role. ${ }^{47}$ Although the numbers studied to date are still small, this approach seems to be promising. In one of these studies, 41 premenopausal women were randomized after antibiotic treatment to receive intravaginal probiotics or placebo twice weekly for 2 weeks and then at the end of each of the next 2 months, ${ }^{75}$ and showed a reduction in UTI recurrence rate by approximately half in the probiotic group (21\% versus $47 \%$ ).

Another study supporting this approach was conducted by Beerepoot et $\mathrm{al}^{16}$ in 252 postmenopausal women with recurrent UTI. After 12 months of prophylaxis with either trimethoprim-sulfamethoxazole or oral capsules containing 109 colony-forming units of L. rhamnosus GR-1 and L. reuteri $\mathrm{RC}-14$, a reduction in recurrences of more than $50 \%$ was seen following both treatments, with median times to the next episode of 6 and 3 months for the antibiotic and probiotic arms, respectively, while resistance following prophylaxis in the antibiotic group nearly doubled. No such increase occurred in the probiotic group. However, not all studies support this approach, because their findings appear to be very variable, depending on the stem used and on the administration methods.

A study conducted by Rönnqvist et al suggested the possibility of using Lactobacillus-impregnated panty liners. Their work showed a reduction in the predisposition of women treated with lactobacilli to develop UTI caused by Group B streptococci, thus demonstrating the feasibility of this method. ${ }^{77}$

As in other fields of medicine, the suggestion of immunization for patients at increased risk of recurrent UTI has been raised. Studies using heat-inactivated strains of uropathogenic E. coli have shown a good response, with good tolerability in patients. ${ }^{78}$ However, heterogeneity of the possible strains causing UTI and our as yet incomplete understanding of the mechanisms underlying bacterial pathogenicity and the host response against them, has prevented licensing of a vaccine for use in patients at risk for recurrent UTI.

Advances in basic research have demonstrated a correlation between the capacity to produce hexameric IgM in response to infection and development of permanent immunity in the organism. ${ }^{79}$ Differences in the subtypes of urothelial receptors have also been investigated and found to play an important role in the development of infection in the host, and are correlated with the degree of disease severity. ${ }^{22}$ Research in this area is still experimental, and further studies are still required to completely understand the mechanisms of infection and immune response, and to develop an efficient prophylactic strategy against recurrent UTI.

\section{Fungal infections}

Consideration should be given to the presence of Candida spp in the urine (candiduria). The finding of Candida in the urine can frequently be the result of contamination during collection of urine samples from patients with Candida resident on the external genitalia. ${ }^{80}$ Candiduria is a condition most often found in elderly, hospitalized, or immunocompromised patients. ${ }^{81}$ Candida albicans is the most common species isolated, accounting for more than half of all cases, followed by Candida glabrata and other Candida spp. ${ }^{14,82}$

Even if asymptomatic, candiduria should be considered as an expression of a serious underlying illness, and predisposing causes should be investigated and treated whenever possible. Identification of the source of candiduria could prove to be useful, but the diagnostic tools at our disposal are currently inadequate to enable correct diagnosis of the site of origin (upper or lower urinary tract, kidneys) that could aid effective treatment. Asymptomatic candiduria in the absence of complicating conditions is not considered to be an indication for treatment. ${ }^{83}$

The mainstay of antibiotic treatment for candiduria is the azolic compounds, mainly fluconazole $200 \mathrm{mg}$ orally daily for 2 weeks. ${ }^{84}$ The use of amphotericin B, which is more toxic, is to be regarded as second-line and for intravesical irrigation in certain settings, because it does treat potential fungal spread to the upper urinary tract (or even worse, systemic spread). ${ }^{85}$

\section{Catheterized patients}

Another important issue is catheter-related UTI in women. Although indwelling catheters are a source of infection in both genders, extraluminal ascending UTI in the female is more common, due to anatomical factors, and again, the 
most common microbes are of fecal origin. Consideration of the role of biofilms surrounding indwelling catheters in long-term catheterized women is therefore necessary. Bacteria become highly resistant to most antibiotics via biofilms, which prevent antibiotics reaching the pathogenic microorganisms. Because adhesion of these microorganisms to catheters is related to the hydrophilicity of the material they are made of, an important first step in the prevention of bacterial colonization and biofilm formation is selection of poorly hydrophilic catheters, such as those made of silicon, and avoidance of latex catheters whenever possible.

Research is presently underway to develop catheters with antimicrobial properties, by using a mixture of antibiotic coatings on the surface of the catheters. ${ }^{86-88}$ Although experimental research has demonstrated good activity of these combinations in preventing colonization, definitive clinical trials demonstrating superiority of medicated over conventional catheters are still lacking. ${ }^{89}$ However, there has been some evidence suggesting the efficacy of medicated catheters for reducing bacteriuria in the short-term setting. ${ }^{90}$

Several antibiotics have been tested for their ability to reduce the risk of biofilm formation. In a recent study by Desai et al application of a nitrofurazone coating showed significant activity against $E$. coli and E. faecalis strains in delaying microbial adherence, which is the first step in biofilm formation. ${ }^{91}$ Other studies have also shown nitrofurazone to be useful against infection by Candida spp. Ciprofloxacinimpregnated catheters were also tested, and similar results were reported for prevention of infection by Gram-negative bacteria during short-time catheterization and for prevention of UTI caused by Pseudomonas aeruginosa. ${ }^{92}$

Other antibiotics investigated in this setting include rifampicin and minocycline, which are currently used in the coating of central venous access systems, and some efforts have been made to extend this application to urinary devices, where they have been shown to reduce the rate of colonization by Gram-positive bacteria, but not the colonization rates for Gram-negative or fungal organisms. ${ }^{90,93}$ Of note, although the efficacy of nitrofurazone-coated catheters has been demonstrated in the short term (less than 1 week), the activity of the rifampicin-minocycline combination has been demonstrated to be superior to no medication for up to 2 weeks. There are currently no data supporting the efficacy of antibacterial coatings on catheters in preventing bacterial colonization or biofilm formation for longer periods of time.

However, antibiotic prophylaxis when using long-term indwelling catheters is not generally recommended due to the unfavorable balance between the advantages and side effects of long-term therapy ${ }^{94}$ and the limited advantages of short-term catheterization in the absence of complicating factors (eg, surgical procedures). ${ }^{95}$ Because no other measures have been shown to have a significant impact on the prevention of UTI in patients with long-term catheters, the recommendation at this time is vigilant monitoring of the urine and prompt initiation of effective therapy as the best way to prevent such complications. ${ }^{96}$

In conclusion, UTI is more difficult to treat in women than in men because of its multifactorial etiology and the limitations posed by the possibility of pregnancy. The first objective for the physician is to identify women at risk for recurrence of UTI as well as to recognize and remove individual risk factors.

\section{Communication with the patient}

As mentioned earlier, recurrent UTI in women, rather than being just a sequence of isolated episodes that can be treated with appropriate antibiotic therapy, is often multifactorial. Thus, the most important step in treatment is identification of the causes leading to the problem. Clearly, communication between the female patient and her physician is paramount, but is not always easy because patients often underestimate their symptoms, or consider them too embarrassing to discuss with their doctor. However, when placed in a specialist setting, women will often appear more uninhibited and more willing to discuss their symptoms.

Subsequently, many patients will delay correct diagnosis and therapeutic strategy, even though the problem could have been easily solved by their family physicians, resulting in higher social and personal costs. It is thus necessary for the physician to spend time speaking with patients reporting UTI symptoms in order to gain a better understanding of the causes of the UTI. Attention must be paid to potential diseases which can work as factors favoring reinfection, because they disturb the normal storage-voiding cycle or reduce body defenses. In particular, any neurologic condition must be taken into account, along with metabolic dysfunction (eg, diabetes), gynecological disorders, and known infectious diseases, eg, human immunodeficiency virus. Detailed inquiry should be made about daily urination habits and any possible changes that could have occurred with time with regard to frequency, urinary volume, and any feelings of discomfort. Particular attention must also be paid to the urination behavior of the female patient, because incorrect hygiene procedures can easily enable passage of enteric bacteria to the female urogenital region, with consequent reinfection. ${ }^{4}$ This aspect, well known to nursing staff, should not be overlooked by the 
general practitioner and must be kept in mind particularly in elderly or less able patients. ${ }^{97}$ Re-education of the patient and/or caregivers can help to prevent or at least reduce the recurrence of UTI.

The use of urinary charts (voiding diaries) could prove useful even in unspecialized settings and help the practitioner to overcome the difficulties of imprecise reporting by patients. These can be presented to the patient and be taken home, in order to give the woman an opportunity to think about her problem in a more comfortable setting and answer questions sincerely and openly.

A subsequent evaluation some days later, together with the results of investigations (such as urinalysis, and culture and sensitivity) will clarify whether the UTI can be adequately treated in general practice, or if there are complications requiring specialist referral. Either way, prescription of broad-spectrum therapy without attempting to identify the causative pathogen should be avoided. General symptoms are not reliable enough to enable diagnosis of UTI without culture and sensitivity analysis..$^{98}$

\section{Disclosure}

The authors report no conflicts of interest in this work.

\section{References}

1. Ronald A. The etiology of urinary tract infection: Traditional and emerging pathogens. Dis Mon. 2003;49(2):71-82.

2. Hummers-Pradier E, Kochen MM. Urinary tract infections in adult general practice patients. Br J Gen Pract. 2002;52(482):752-761.

3. Hill MJ, Drasar BS. The normal colonic bacterial flora. Gut. 1975; 16(4):318-323.

4. Yamamoto S, Tsukamoto T, Terai A, Kurazono H, Takeda Y, Yoshida O. Genetic evidence supporting the fecal-perineal-urethral hypothesis in cystitis caused by Escherichia coli. J Urol. 1997;157(3):1127-1129.

5. Polito M, Minardi D, Montnari MP, Varaldo PE. Adherence of Gram negative uropathogens to human uroepithelial cells. Eur Urol. 1987; 13(1-2):74-78.

6. Plos K, Lomberg H, Hull S, Johansson I, Svanborg C. Escherichia coli in patients with renal scarring: Genotype and phenotype of Gal alpha 1-4Gal beta-, Forssman- and mannose-specific adhesins. Pediatr Infect Dis J. 1991;10(1):15-19.

7. Ab Hadi I, Bliss RD, Lennard TW, Welch AR. Primary squamous cell carcinoma of the thyroid gland: A case report and role of radiotherapy. Surgeon. 2007;5(4):249-251.

8. Zhou G, Mo WJ, Sebbel P, et al. Uroplakin Ia is the urothelial receptor for uropathogenic Escherichia coli: Evidence from in vitro FimH binding. J Cell Sci. 2001;114(Pt 22):4095-4103.

9. Mulvey MA, Schilling JD, Martinez JJ, Hultgren SJ. Bad bugs and beleaguered bladders: Interplay between uropathogenic Escherichia coli and innate host defenses. Proc Natl Acad Sci U SA. 2000;97(16): 8829-8835.

10. Bishop BL, Duncan MJ, Song J, Li G, Zaas D, Abraham SN. Cyclic AMP-regulated exocytosis of Escherichia coli from infected bladder epithelial cells. Nat Med. 2007;13(5):625-630.

11. Rupp ME, Soper DE, Archer GL. Colonization of the female genital tract with Staphylococcus saprophyticus. J Clin Microbiol. 1992;30(11): 2975-2979.
12. Wagenlehner FM, Niemetz AH, Weidner W, Naber KG. Spectrum and antibiotic resistance of uropathogens from hospitalised patients with urinary tract infections: 1994-2005. Int J Antimicrob Agents. 2008; 31(Suppl 1):S25-S34.

13. Marcus RJ, Post JC, Stoodley P, et al. Biofilms in nephrology. Expert Opin Biol Ther. 2008;8(8):1159-1166.

14. Kauffman CA, Vazquez JA, Sobel JD, et al. Prospective multicenter surveillance study of funguria in hospitalized patients. The National Institute for Allergy and Infectious Diseases (NIAID) Mycoses Study Group. Clin Infect Dis. 2000;30(1):14-18.

15. Rivett AG, Perry JA, Cohen J. Urinary candidiasis: A prospective study in hospitalized patients. Urol Res. 1986;14(3):153-173.

16. Sobel JD, Vazquez JA. Fungal infections of the urinary tract. World $J$ Urol. 1999;17(6):410-414.

17. Beutler B, Moresco EM. The forward genetic dissection of afferent innate immunity. Curr Top Microbiol Immunol. 2008;321:3-26.

18. Fischer H, Yamamoto M, Akira S, Beutler B, Svanborg C. Mechanism of pathogen-specific TLR4 activation in the mucosa: Fimbriae, recognition receptors and adaptor protein selection. Eur J Immunol. 2006;36(2): 267-277.

19. Bergsten G, Samuelsson M, Wullt B, Leijonhufvud I, Fischer H, Svanborg C. PapG-dependent adherence breaks mucosal inertia and triggers the innate host response. J Infect Dis. 2004;189(9):1734-1742.

20. Ragnarsdottir B, Samuelsson M, Gustafsson MC, Leijonhufvud I, Karpman D, Svanborg C. Reduced toll-like receptor 4 expression in children with asymptomatic bacteriuria. J Infect Dis. 2007;196(3): 475-484.

21. Benson M, Jodal U, Agace W, et al. Interleukin (IL)-6 and IL-8 in children with febrile urinary tract infection and asymptomatic bacteriuria. J Infect Dis. 1996;174(5):1080-1084.

22. Nielubowicz GR, Mobley HL. Host-pathogen interactions in urinary tract infection. Nat Rev Urol. 2010;7(8):430-441.

23. Frendeus B, Godaly G, Hang L, Karpman D, Svanborg C. Interleukin-8 receptor deficiency confers susceptibility to acute pyelonephritis. $J$ Infect Dis. 2001;183(Suppl 1):S56-S60.

24. Lundstedt AC, McCarthy S, Gustafsson MC, et al. A genetic basis of susceptibility to acute pyelonephritis. PLoS One. 2007;2(9):e825.

25. Mitsumori K, Terai A, Yamamoto S, Yoshida O. Virulence characteristics and DNA fingerprints of Escherichia coli isolated from women with acute uncomplicated pyelonephritis. J Urol. 1997;158(6):2329-2332.

26. Hooton TM, Stapleton AE, Roberts PL, et al. Perineal anatomy and urine-voiding characteristics of young women with and without recurrent urinary tract infections. Clin Infect Dis. 1999;29(6):1600-1601.

27. Aroutcheva A, Gariti D, Simon M, et al. Defense factors of vaginal lactobacilli. Am J Obstet Gynecol. 2001;185(2):375-379.

28. Kirjavainen PV, Pautler S, Baroja ML, et al. Abnormal immunological profile and vaginal microbiota in women prone to urinary tract infections. Clin Vaccine Immunol. 2009;16(1):29-36.

29. Barrons R, Tassone D. Use of Lactobacillus probiotics for bacterial genitourinary infections in women: A review. Clin Ther. 2008;30(3):453-468.

30. Raz R. Postmenopausal women with recurrent UTI. Int J Antimicrob Agents. 2001;17(4):269-271.

31. Hooton TM, Scholes D, Stapleton AE, et al. A prospective study of asymptomatic bacteriuria in sexually active young women. $N$ Engl $J$ Med. 2000;343(14):992-997.

32. Wullt B, Ragnarsdottir B, Fischer H, Gronberg JH, Lutay N, Svanborg C. Urogenital Infections: EAU- International Consultation on Urological Diseases; 2010.

33. Hawn TR, Scholes D, Wang H, et al. Genetic variation of the human urinary tract innate immune response and asymptomatic bacteriuria in women. PLoS One. 2009;4(12):e8300.

34. Hawn TR, Scholes D, Li SS, et al. Toll-like receptor polymorphisms and susceptibility to urinary tract infections in adult women. PLoS One. 2009;4(6):e5990.

35. Dobrindt U, Blum-Oehler G, Hartsch T, et al. S-Fimbria-encoding determinant sfa(I) is located on pathogenicity island III(536) of uropathogenic Escherichia coli strain 536. Infect Immun. 2001;69(7):4248-4256. 
36. Ziegler T, Jacobsohn N, Funfstuck R. Correlation between blood group phenotype and virulence properties of Escherichia coli in patients with chronic urinary tract infection. Int $J$ Antimicrob Agents. 2004; 24(Suppl 1):S70-S75.

37. Kinane DF, Blackwell CC, Brettle RP, Weir DM, Winstanley FP, Elton RA. ABO blood group, secretor state, and susceptibility to recurrent urinary tract infection in women. Br Med J (Clin Res Ed). 1982; 285(6334):7-9.

38. Hooton TM. Recurrent urinary tract infection in women. Int J Antimicrob Agents. 2001;17(4):259-268.

39. Haylen BT, Lee J, Husselbee S, Law M, Zhou J. Recurrent urinary tract infections in women with symptoms of pelvic floor dysfunction. Int Urogynecol J. 2009;20(7):837-842.

40. Patterson TF, Andriole VT. Detection, significance, and therapy of bacteriuria in pregnancy. Update in the managed health care era. Infect Dis Clin North Am. 1997;11(3):593-608.

41. Hill JB, Sheffield JS, McIntire DD, Wendel GD Jr. Acute pyelonephritis in pregnancy. Obstet Gynecol. 2005;105(1):18-23.

42. Golan A, Wexler S, Amit A, Gordon D, David MP. Asymptomatic bacteriuria in normal and high-risk pregnancy. Eur J Obstet Gynecol Reprod Biol. 1989;33(2):101-108.

43. Whalley P. Bacteriuria of pregnancy. Am J Obstet Gynecol. 1967;97(5): 723-738.

44. Krcmery S, Hromec J, Demesova D. Treatment of lower urinary tract infection in pregnancy. Int J Antimicrob Agents. 2001;17(4):279-282.

45. Nygaard IE, Johnson JM. Urinary tract infection in elderly women. Am Fam Physician 1996;53(1):175-182.

46. Raz R, Gennesin Y, Wasser J, et al. Recurrent urinary tract infections in postmenopausal women. Clin Infect Dis. 2000;30(1):152-156.

47. Raz R, Stamm WE. A controlled trial of intravaginal estriol in postmenopausal women with recurrent urinary tract infections. $N$ Engl $J$ Med. 1993;329(11):753-756.

48. Nicolle LE. Asymptomatic bacteriuria in the elderly. Infect Dis Clin North Am. 1997;11(3):647-662.

49. Geerlings SE, Stolk RP, Camps MJ, et al. Asymptomatic bacteriuria may be considered a complication in women with diabetes. Diabetes Mellitus Women Asymptomatic Bacteriuria Utrecht Study Group. Diabetes Care. 2000;23(6):744-749.

50. Geerlings SE, Stolk RP, Camps MJ, Netten PM, Collet TJ, Hoepelman AI. Risk factors for symptomatic urinary tract infection in women with diabetes. Diabetes Care. 2000;23(12):1737-1741.

51. Messelink B, Benson T, Berghmans B, et al. Standardization of terminology of pelvic floor muscle function and dysfunction: Report from the pelvic floor clinical assessment group of the International Continence Society. Neurourol Urodyn. 2005;24(4):374-380.

52. Carlson KV, Rome S, Nitti VW. Dysfunctional voiding in women. J Urol. 2001;165(1):143-147.

53. Yagci S, Kibar Y, Akay O, et al. The effect of biofeedback treatment on voiding and urodynamic parameters in children with voiding dysfunction. J Urol. 2005;174(5):1994-1997.

54. Kuo HC. Videourodynamic characteristics and lower urinary tract symptoms of female bladder outlet obstruction. Urology. 2005;66(5): 1005-1009.

55. Minardi D, Parri G, d'Anzeo G, Fabiani A, El Asmar Z, Muzzonigro G. Perineal ultrasound evaluation of dysfunctional voiding in women with recurrent urinary tract infections. $J$ Urol. 2008;179(3):947-951.

56. Scholes D, Hooton TM, Roberts PL, Stapleton AE, Gupta K, Stamm WE. Risk factors for recurrent urinary tract infection in young women. J Infect Dis. 2000;182(4):1177-1182.

57. Farage M, Bramante M, Otaka Y, Sobel J. Do panty liners promote vulvovaginal candidiasis or urinary tract infections? A review of the scientific evidence. Eur J Obstet Gynecol Reprod Biol. 2007;132(1):8-19.

58. Smaill F, Vazquez JC. Antibiotics for asymptomatic bacteriuria in pregnancy. Cochrane Database Syst Rev. 2007;2:CD000490.

59. Grabe M, Bergland-Johansen TE, Botto H, et al. Guidelines on urological infections. Presented at the 25th European Association of Urology Annual Congress. Barcelona, Spain, April 16-20, 2010.
60. Kahlmeter G. An international survey of the antimicrobial susceptibility of pathogens from uncomplicated urinary tract infections: The ECO. SENS Project. J Antimicrob Chemother. 2003;51(1):69-76.

61. Bjerrum L, Gahrn-Hansen B, Grinsted P. Pivmecillinam versus sulfamethizole for short-term treatment of uncomplicated acute cystitis in general practice: A randomized controlled trial. Scand J Prim Health Care. 2009;27(1):6-11.

62. Schito GC, Gualco L, Naber KG, et al. Do different susceptibility breakpoints affect the selection of antimicrobials for treatment of uncomplicated cystitis? J Chemother. 2010;22(5):345-354.

63. Bean DC, Krahe D, Wareham DW. Antimicrobial resistance in community and nosocomial Escherichia coli urinary tract isolates, London 2005-2006. Ann Clin Microbiol Antimicrob. 2008;7:13.

64. Schito GC, Naber KG, Botto H, et al. The ARESC study: An international survey on the antimicrobial resistance of pathogens involved in uncomplicated urinary tract infections. Int J Antimicrob Agents. 2009; 34(5):407-413

65. Christensen $\mathrm{B}$. Which antibiotics are appropriate for treating bacteriuria in pregnancy? J Antimicrob Chemother. 2000;46(Suppl A):29-34.

66. Briggs GG, Freeman RK, Yaffe SJ. Drugs in Lactation. 2nd ed. Philadelphia, PA: Lippicott, Williams \& Wilkins; 2002.

67. Minardi D, d'Anzeo G, Parri G, et al. The role of uroflowmetry biofeedback and biofeedback training of the pelvic floor muscles in the treatment of recurrent urinary tract infections in women with dysfunctional voiding: A randomized controlled prospective study. Urology. 2010;75(6):1299-1304.

68. Costantini E, Lazzeri M, Bini V, et al. Open-label, longitudinal study of tamsulosin for functional bladder outlet obstruction in women. Urol Int. 2009;83(3):311-315.

69. Ludwig M, Hoyme U, Weidner W. Recurrent urinary tract infection in women. Long-term antibiotic prophylaxis. Urologe A. 2006;45(4): 436-442. German.

70. Perez-Lopez FR, Haya J, Chedraui P. Vaccinium macrocarpon: An interesting option for women with recurrent urinary tract infections and other health benefits. J Obstet Gynaecol Res. 2009;35(4): 630-639.

71. Howell AB. Bioactive compounds in cranberries and their role in prevention of urinary tract infections. Mol Nutr Food Res. 2007;51(6): 732-737.

72. Howell AB, Botto H, Combescure C, et al. Dosage effect on uropathogenic Escherichia coli anti-adhesion activity in urine following consumption of cranberry powder standardized for proanthocyanidin content: A multicentric randomized double blind study. BMC Infect Dis. 2010;10:94.

73. Efros M, Bromberg W, Cossu L, Nakeleski E, Katz AE. Novel concentrated cranberry liquid blend, UTI-STAT with Proantinox, might help prevent recurrent urinary tract infections in women. Urology. 2010; 76(4):841-845.

74. Barbosa-Cesnik C, Brown MB, Buxton M, Zhang L, Debusscher J, Foxman B. Cranberry juice fails to prevent recurrent urinary tract infection: Results from a randomized placebo-controlled trial. Clin Infect Dis. 2011;52(1):23-30.

75. Reid G, Bruce AW, Taylor M. Influence of three-day antimicrobial therapy and lactobacillus vaginal suppositories on recurrence of urinary tract infections. Clin Ther. 1992;14(1):11-16.

76. Beerepoot MAJTG, Nys S, Vanderwal WM, et al. Lactobacillus rhamnosus GR-1 and L. reuteri $\mathrm{RC}-14$ versus trimethoprim-sulfamethoxazole (TMP/SMX) in the prevention of recurrent urinary tract infections (rUTIs) in postmenopausal women: A randomized double-blind noninferiority trial. ICAAC. 2009:Abstract L1-1656a.

77. Ronnqvist PD, Forsgren-Brusk UB, Grahn-Hakansson EE. Lactobacilli in the female genital tract in relation to other genital microbes and vaginal pH. Acta Obstet Gynecol Scand. 2006;85(6): 726-735.

78. Grischke EM, Ruttgers H. Treatment of bacterial infections of the female urinary tract by immunization of the patients. Urol Int. 1987;42(5): $338-341$. 
79. Petrusic V, Stojanovic M, Zivkovic I, Inic-Kanada A, Dimitrijevic L. Changes in composition of IgM polymers in patients suffering from recurrent urinary bacterial infections after bacterial immunization treatment. Immunol Invest. 2010;39(8):781-795.

80. Malani AN, Kauffman CA. Candida urinary tract infections: Treatment options. Expert Rev Anti Infect Ther. 2007;5(2):277-284.

81. Carvalho M, Guimaraes CM, Mayer JR Jr, Bordignon GP, Queiroz-Telles F. Hospital-associated funguria: Analysis of risk factors, clinical presentation and outcome. Braz J Infect Dis. 2001;5(6):313-318.

82. Chen SC, Tong ZS, Lee OC, et al. Clinician response to Candida organisms in the urine of patients attending hospital. Eur J Clin Microbiol Infect Dis. 2008;27(3):201-208.

83. Pappas PG, Kauffman CA, Andes D, et al. Clinical practice guidelines for the management of candidiasis: 2009 update by the Infectious Diseases Society of America. Clin Infect Dis. 2009;48(5):503-535.

84. Sobel JD, Fisher J, Kauffman CA. Guidelines for the treatment of fungal urinary tract infections. Urinogenital Infections: EAU- International Consultation on Urological Diseases; 2010.

85. Tuon FF, Amato VS, Penteado Filho SR. Bladder irrigation with amphotericin B and fungal urinary tract infection - systematic review with meta-analysis. Int J Infect Dis. 2009;13(6):701-706.

86. Gaonkar TA, Caraos L, Modak S. Efficacy of a silicone urinary catheter impregnated with chlorhexidine and triclosan against colonization with Proteus mirabilis and other uropathogens. Infect Control Hosp Epidemiol. 2007;28(5):596-598.

87. Kowalczuk D, Ginalska G, Golus J. Characterization of the developed antimicrobial urological catheters. Int J Pharm. 2010;402(1-2):175-183.

88. Darouiche RO, Mansouri MD, Gawande PV, Madhyastha S. Antimicrobial and antibiofilm efficacy of triclosan and Dispersin B combination. J Antimicrob Chemother. 2009;64(1):88-93.
89. Jahn P, Preuss M, Kernig A, Seifert-Huhmer A, Langer G. Types of indwelling urinary catheters for long-term bladder drainage in adults. Cochrane Database Syst Rev. 2007(3):CD004997.

90. Schumm K, Lam TB. Types of urethral catheters for management of short-term voiding problems in hospitalised adults. Cochrane Database Syst Rev. 2008(2):CD004013.

91. Desai DG, Liao KS, Cevallos ME, Trautner BW. Silver or nitrofurazone impregnation of urinary catheters has a minimal effect on uropathogen adherence. J Urol. 2010;184(6):2565-2571.

92. Reid G, Sharma S, Advikolanu K, Tieszer C, Martin RA, Bruce AW. Effects of ciprofloxacin, norfloxacin, and ofloxacin on in vitro adhesion and survival of Pseudomonas aeruginosa AK1 on urinary catheters. Antimicrob Agents Chemother. 1994;38(7):1490-1495.

93. Darouiche RO, Smith JA Jr, Hanna H, et al. Efficacy of antimicrobialimpregnated bladder catheters in reducing catheter-associated bacteriuria: A prospective, randomized, multicenter clinical trial. Urology. 1999;54(6):976-981.

94. Niel-Weise BS, van den Broek PJ. Urinary catheter policies for long-term bladder drainage. Cochrane Database Syst Rev. 2005(1):CD004201.

95. Niel-Weise BS, van den Broek PJ. Antibiotic policies for short-term catheter bladder drainage in adults. Cochrane Database Syst Rev. 2005(3):CD005428.

96. Willson M, Wilde M, Webb ML, et al. Nursing interventions to reduce the risk of catheter-associated urinary tract infection: Part 2: Staff education, monitoring, and care techniques. J Wound Ostomy Continence Nurs. 2009;36(2):137-154.

97. Wang K, Palmer MH. Women's toileting behaviour related to urinary elimination: Concept analysis. J Adv Nurs. 2010;66(8):1874-1884.

98. Kuklinski D, Koduri S. Predicting urinary tract infections in a urogynecology population. Urol Nurs. 2008;28(1):56-60.
International Journal of General Medicine

\section{Publish your work in this journal}

The International Journal of General Medicine is an international, peer-reviewed open-access journal that focuses on general and internal medicine, pathogenesis, epidemiology, diagnosis, monitoring and treatment protocols. The journal is characterized by the rapid reporting of reviews, original research and clinical studies across all disease areas.

\section{Dovepress}

A key focus is the elucidation of disease processes and management protocols resulting in improved outcomes for the patient.The manuscript management system is completely online and includes a very quick and fair peer-review system. Visit http://www.dovepress.com/ testimonials.php to read real quotes from published authors. 